\title{
A LIMIT THEOREM FOR A FUNCTION OF THE INCREMENTS OF A DECOMPOSABLE PROCESS
}

\author{
BY \\ ROBERT COGBURN AND HOWARD G. TUCKER(1)
}

1. Introduction and summary. Let $X_{T}$ be a separable, continuous (in law), decomposable process on a finite time interval $T=\left[t_{1}, t_{2}\right]$, that is, the increments $X_{[s, t)}=X_{t}-X_{s}$ over disjoint intervals in $T$ are independent random variables and $X_{\left[t_{1}, t\right)}$ is continuous in law, hence almost surely (a.s.): $X_{t} \rightarrow X_{\text {s }}$ a.s. as $t \rightarrow$ for each $s \in T$. For such process the famous Raikov theorem states that if $E X_{\left[t_{1}, t\right)}=0$ for each $t$, then $X_{\left[t_{1}, t_{2}\right)}$ is distributed $\mathfrak{N}\left(0, \sigma^{2}\right)$ if, and only if, $\sum_{k} X_{n k}^{2} \rightarrow \sigma^{2}$ in probability as $n \rightarrow \infty$, where $X_{n k}=X_{\left(t_{n, k-1}, t_{n k}\right)}$ and $\sum\left[t_{n, k-1}, t_{n k}\right)=T$ are partitions, $\mathcal{P}_{n}(T)$, of $T$ with $\max _{k}\left(t_{n k}-t_{n, k-1}\right) \rightarrow 0$. Paul Lévy then obtained for the Brownian motion process that, if the partitions $\left\{P_{n}(T)\right\}$ are a sequence ordered by refinement with $\max \left(t_{n k}-t_{n, k-1}\right) \rightarrow 0$, then in fact $\sum X_{n k}^{2} \rightarrow \sigma^{2}$ a.s., the variance of $X_{\left[t_{1}, t_{2}\right)}$. The Brownian motion assumption is easily replaced by the assumption that the process is continuous and the increments are normally distributed and centered at expectations.

Recently M. Loève [2] considered the problem for more general functions of the increments, $g\left(X_{n k}\right)$, than $X_{n k}^{2}$. His results concern the general double sequences of independent, uniformly asymptotically negligible random variables of the modern central limit problem. When applied to processes, these results generalize the Raikov theorem to yield convergence in law of $\sum g\left(X_{n k}\right)$ when $g$ is a continuous function having a second derivative at the origin with $g(0)=0$. The second derivative assumption can be weakened, but then centering constants have to be introduced to obtain convergence.

Finally Rubin and Tucker [3] generalized the Paul Lévy theorem (for squared increments), showing that a.s. convergence held for separable, decomposable processes with stationary increments provided one took partitions with equal increments and the number of elements, $k_{n}$, in the $n$th partition went to infinity very rapidly, namely $\sum\left(1 / k_{n}\right)^{1 / 3}<\infty$.

We will first drop the stationary increments assumption and show that the sum of squared increments converges a.s. along the sequence $\left\{P_{n}(T)\right\}$, thus

This paper has been submitted to and accepted for publication by the Proceedings of the American Mathematical Society. It has been transferred to these Transactions, with the consent of the authors, for technical reasons. Presented to the Society, January 23, 1961; received by the editors June 24, 1960 and, in revised form, September 23, 1960.

(1) This investigation was supported (in part) by a research grant (No. RG-3666) from the National Institutes of Health, Public Health Service, and with the partial support of the Office of Naval Research (Nonr-222-43). This paper in whole or in part may be reproduced for any purpose of the United States Government. 
improving the result of Rubin and Tucker. Then we will show that the result extends to continuous functions, $g$, of the increments having second derivatives at the origin, paralleling the extension of Raikov's theorem by Loève.

2. Some lemmas. In the sequel, $X_{T}, Y_{T}$, and $Z_{T}$, with or without primes, denote separable, continuous, decomposable processes on $T$. For simplicity we take $T=[0,1]$ and $\left\{\rho_{n}(T)\right\}$ denotes a sequence of partitions of $T$ ordered by refinement with $\max \left\{t_{n, k}-t_{n, k-1} \mid 1 \leqq k \leqq k_{n}\right\} \rightarrow 0$ as $n \rightarrow \infty$. Adding some partitions to the sequence if necessary, we can assume without loss of generality that the $n$th partition divides $T$ into exactly $n$ increments. Unless otherwise stated, all limits will be along $\left\{\rho_{n}(T)\right\}$. The truncation of a random variable at $b>0$ will be denoted by $X^{(b)}=X I_{[|X| \leqq}$.

The characteristic function of $X_{(0, t)}$ then has the form $f_{t}=\exp \left\{\psi_{t}\right\}$ where, in standard notation

$$
\psi_{t}(u)=i u \alpha_{t}+\int\left(e^{i u x}-1-\frac{i u x}{1+x^{2}}\right) \frac{1+x^{2}}{x^{2}} d \Psi_{t}(x)
$$

and will be denoted simply $\left(\alpha_{t}, \Psi_{t}\right)$. The law of the process over $T$ will be denoted by $\left(\alpha_{T}, \Psi_{T}\right)$.

For the reader who is unfamiliar with this formalism we may remark that for a decomposable process, neglecting a null set of sample functions, the sample functions are bounded, have right and left limits at every point, and the discontinuities are jumps. Furthermore, in any time interval $[0, t)$, the number of jumps of the sample function whose magnitude lies in $(-\infty, x)$ for $x<0$ or $(x, \infty)$ for $x>0$ is finite and Poisson distributed. Letting $L_{t}(x)$ denote the expected number of jumps in time interval $[0, t)$ whose magnitude is $\left\langle x<0\right.$ and $-L_{t}(x)$ denote the expected number of jumps $\geqq x>0$, we have

$$
\begin{aligned}
\Psi_{t}(x) & =\int_{-\infty}^{x} \frac{y^{2}}{1+y^{2}} d L_{t}(y), & & x<0, \\
\Psi_{t}(\infty)-\Psi_{t}(x) & =\int_{x}^{\infty} \frac{y^{2}}{1+y^{2}} d L_{t}(y), & & x>0 .
\end{aligned}
$$

Finally, $\Psi(0+)-\Psi(0-)=\sigma^{2}$ is the variance of the normal part of the process, and $\alpha_{t}$ corresponds to a sure function or "drift" on $T$.

Our assumptions imply that $\alpha_{t}$ is a continuous function of $t$. In addition we assume $\alpha_{t}$ is of bounded variation on $T$ in what follows. All summations will be for $k=1,2, \cdots, n$, unless otherwise stated.

LEMмA 1. The sum $\Gamma_{n}=\sum w_{n k} X_{n k}$ is uniformly bounded in probability for all partitions of $T$ and constants $w_{n k}$ with $\left|w_{n k}\right| \leqq 1$, i.e., for arbitrary $\epsilon>0$, there is a constant $b_{\epsilon}$ depending only on $\epsilon$ such that $P\left[\left|\Gamma_{n}\right|>b_{\epsilon}\right]<\epsilon$.

Proof. Let $X_{T}$ have law $\left(\alpha_{T}, \Psi_{T}\right)$, and let $\pm b$ be continuity points of $\Psi_{1}(x)$. Define $V_{T}$ by 


$$
Y_{t}=X_{t}-J_{b, t}
$$

where $J_{b, t}$ is the sum of the (finite number of) jumps of the sample function $X_{t}$ during $[0, t)$ whose absolute magnitude exceeds $b$. It is obvious that $Y_{T}$ is a separable, continuous, decomposable process with law $\left(\beta_{T}, \Psi_{T}^{b}\right)$ where

$$
\beta_{t}=\alpha_{t}-\int_{|x|>b} \frac{1}{x} d \Psi_{t}(x), \quad \Psi_{t}^{b}(x)=\left\{\begin{array}{lr}
\Psi_{t}(-b) & \text { if } x \leqq b, \\
\Psi_{t}(x) & \text { if }-b<x \leqq b \\
\Psi_{t}(b) & \text { if } x>b .
\end{array}\right.
$$

Let $\Lambda_{n}=\sum w_{n k} Y_{n k}$. The process $Y_{T}$ has finite moments and for all partitions

$$
\left|E \Lambda_{n}\right| \leqq \text { Total variation }\left\{\beta_{t} ; 0 \leqq t \leqq 1\right\}+b \Psi_{1}[-b, b]
$$

and

$$
\operatorname{Var}\left(\Lambda_{n}\right)=\sum w_{n k}^{2} \operatorname{Var}\left(Y_{n k}\right) \leqq \operatorname{Var}\left(Y_{1}\right)<\infty .
$$

By Chebishev's inequality, the $\Lambda_{n}-E \Lambda_{n}$, hence the $\Lambda_{n}$ are uniformly bounded in probability. Now let $A=\left[\sup \left\{\left|X_{t}\right| ; 0 \leqq t \leqq 1\right\} \leqq b / 2\right], B=\left[\left|\Lambda_{n}\right| \leqq b_{\epsilon}\right]$ and $C=\left[\left|\Gamma_{n}\right| \leqq b_{\epsilon}\right]$. Since $A B=A C$, we obtain $\left|P B^{c}-P C^{c}\right| \leqq P A^{c}$. Letting $b, b_{\epsilon}$ be large enough so that $P A^{c}<\epsilon / 2$ and $P B^{c}<\epsilon / 2$ for all partitions completes the proof of the lemma.

Lemma 2. Let $Y_{T}$ and $Z_{T}$ be independent processes and $V_{n}=\sum Y_{n k} Z_{n k}$. Then, letting $P^{z}$ denote the conditional probability given $Z_{T}, P^{z}\left[\left|V_{n}\right|>\epsilon\right] \rightarrow 0$ a.s. for each $\epsilon>0$.

Proof. Let $z_{t}$ be a sure function on $T$ having right and left limits at every point, jump discontinuities and at most a finite number of jumps exceeding any $\delta>0$ in absolute value. Set

$$
\begin{aligned}
\sum z_{n k} Y_{n k} & =L_{\delta, n}+M_{\delta, n} \text { where } \\
L_{\delta, n} & =\sum_{\left\{k ;\left|z_{n k}\right|>\delta\right\}} z_{n k} Y_{n k} .
\end{aligned}
$$

Then the almost sure continuity of $Y_{T}$ yields $L_{\delta, n} \rightarrow 0$ in probability (in fact, $L_{\delta, n} \rightarrow 0$ a.s.). On the other hand,

$$
M_{\delta, n}=\delta \sum w_{n k} Y_{n k} \text { where } w_{n k}=z_{n k} / \delta \text { or } 0
$$

according as $\left|z_{n k}\right| \leqq \delta$ or $\left|z_{n k}\right|>\delta$. By Lemma $1, P\left[\left|M_{\delta, n}\right|>\epsilon / 2\right]$ $=P\left[\left|\sum w_{n k} Y_{n k}\right|>\epsilon / 2 \delta\right] \rightarrow 0$ uniformly in $n$ as $\delta \rightarrow 0$.

The conditional law of $Y_{T}$ given $Z_{T}$ can, by independence, be taken to be the law of $Y_{T}$. Since outside a null set the sample functions $Z_{t}(\omega)$ satisfy the assumptions on $z_{t}$, we can replace $z_{t}$ by $Z_{t}(\omega)$ in what precedes and obtain

$$
P^{z}\left[\left|V_{n}\right|>\epsilon\right] \leqq P^{z}\left[\left|L_{\delta, n}\right|>\epsilon / 2\right]+P^{z}\left[\left|M_{\delta, n}\right|>\epsilon / 2\right] \text { a.s. }
$$


Letting $n \rightarrow \infty$ and then $\delta \rightarrow 0$ proves the lemma.

Lemma 3. Let $X_{T}, Y_{T}$, and $Z_{T}$ be three independent processes, $U_{n}=\sum X_{n k} Z_{n k}$, $V_{n}=\sum Y_{n k} Z_{n k}$ and $W_{n}=U_{n}-V_{n}$. Then

$$
W_{n} \stackrel{\text { a.s. }}{\longrightarrow} 0 \text { implies } U_{n} \stackrel{\text { a.s. }}{\longrightarrow} 0 \text { and } V_{n} \stackrel{\text { a.s. }}{\longrightarrow} 0 .
$$

Proof. Let $\epsilon>0$ be arbitrary and observe that

$$
\left[\left|U_{n}\right|>2 \epsilon\right]\left[\left|V_{n}\right|<\epsilon\right] \subset\left[\left|W_{n}\right|>\epsilon\right] .
$$

Let $A_{n}=\left[\left|U_{n}\right|>2 \epsilon\right]$ and $B_{m m}=A_{m}, B_{m n}=A_{m}^{c} A_{m+1}^{c} \cdots A_{n-1}^{c} A_{n}$ for $n>m$. Then $U_{n \geq m} A_{n}-U_{n \geq m} B_{m n}\left[\left|V_{n}\right| \geqq \epsilon\right] \subset \bigcup_{n \geq m}\left[\left|W_{n}\right|>\epsilon\right]$. Since $U_{n}$ and $V_{n}$ are conditionally independent given $Z_{T}$ we have

$$
P^{z}\left\{\bigcup_{n \geq m}^{\bigcup} B_{m n}\left[\left|V_{n}\right| \geqq \epsilon\right]\right\}=\sum_{n \geq m} P^{Z} B_{m n} P^{Z}\left[\left|V_{n}\right| \geqq \epsilon\right] \text { a.s. }
$$

By Lemma 2, given $\delta>0$ we can find a set $C$ and an $m$ such that

$$
P^{z}\left[\left|V_{n}\right| \geqq \epsilon\right]<\frac{1}{2} \text { on } C \text { for all } n \geqq m \text { and } P C^{c}<\delta .
$$

Then

$$
\begin{aligned}
E\left(\sum_{n \geq m} P^{z} B_{m n} P^{z}\left[\left|V_{n}\right| \geqq \epsilon\right]\right) & \leqq \frac{1}{2} \sum_{n \geq m} P B_{m n} C+\sum_{n \geq m} P B_{m n} C^{c} \\
& \leqq \frac{1}{2} P \underset{n \geq m}{\bigcup} A_{n}+\delta
\end{aligned}
$$

hence,

$$
\frac{1}{2} P \underset{n \geq m}{\cup} A_{n} \leqq P \bigcup_{n \geq m}\left[\left|W_{n}\right|>\epsilon\right]+\delta .
$$

Again, the lemma follows upon letting $n \rightarrow \infty$ and then $\delta \rightarrow 0$.

LeMma 4. Let $\left\{U_{n}\right\}$ and $\left\{V_{n}\right\}$ be any two sequences of random variables which are independent of each other and have the same joint distributions. If $W_{n}=U_{n}+V_{n} \rightarrow W$ a.s., then $U_{n} \rightarrow U$ a.s. and $V_{n} \rightarrow V$ a.s. where $U$ and $V$ are independent and identically distributed and $U+V=W$.

Proof. Since

$$
\begin{aligned}
{\left[U_{n}-U_{m}>\epsilon\right]\left[V_{n}-V_{m}>\epsilon\right] } & \cup\left[U_{m}-U_{n}>\epsilon\right]\left[V_{m}-V_{n}>\epsilon\right] \\
\subset & \subset\left[\left|W_{n}-W_{m}\right|>2 \epsilon\right],
\end{aligned}
$$

we have 


$$
P^{2}\left[V_{n}-V_{m}>\epsilon\right]+P^{2}\left[V_{m}-V_{n}>\epsilon\right] \leqq P\left[\left|W_{n}-W_{m}\right|>2 \epsilon\right] \rightarrow 0
$$

as $n, m \rightarrow \infty$, hence $P\left[\left|V_{n}-V_{m}\right|>\epsilon\right] \rightarrow 0$. Also,

$$
\bigcup_{n \geq m}\left[\left|U_{n}-U_{m}\right|>2 \epsilon\right]\left[\left|V_{n}-V_{m}\right| \leqq \epsilon\right] \subset \underset{n \geq m}{\bigcup}\left[\left|W_{n}-W_{m}\right|>\epsilon\right]
$$

and, by the lemma for events (Loève $[1$, p. 246]),

$$
\begin{aligned}
\left(\inf _{n \geq m} P\left[\left|V_{n}-V_{m}\right| \leqq \epsilon\right]\right) P \underset{n \geq m}{\cup}\left[\left|U_{n}-U_{m}\right|\right. & >2 \epsilon] \\
& \leqq P \underset{n \geq m}{\cup}\left[\left|W_{n}-W_{m}\right|>\epsilon\right]
\end{aligned}
$$

It follows that $P \bigcup_{n \geq m}\left[\left|U_{n}-U_{m}\right|>2 \epsilon\right] \rightarrow 0$ as $m \rightarrow \infty$, and the conclusion of the lemma is immediate.

3. Two limit theorems. The proofs of the following theorems depend upon repeated application of the methods of symmetrization and truncation, leading to the use of the martingale theorems.

Theorem 1. Let $X_{T}$ be a separable, continuous, decomposable process with law $\left(\alpha_{T}, \Psi_{T}\right)$, where $\alpha_{t}$ is a function of bounded variation on $T$. Then along the sequence of successive refinements, $\otimes_{n}(T)$, of $T$ with the length of the largest interval converging to 0 ,

$$
\int_{T}\left(d X_{t}\right)^{2}=\lim _{n \rightarrow \infty} \sum X_{n k}^{2}=\sigma^{2}+\sum J_{t}^{2} \quad \text { a.s. }
$$

where $\sigma^{2}$ is the variance of the normal component of $X_{\left[t_{1}, t_{2}\right)}$ and $\sum J_{t}^{2}$ is the sum of the squares of the jumps of $X_{T}$.

Proof. The proof is divided into three parts.

1. Let $X_{T}$ and $X_{T}^{\prime}$ be independent, identically distributed processes with symmetric distributions. Recall the definition of $J_{b, t}$ in Lemma 1 , introduce the curtailed process $\bar{X}_{t}^{b}=X_{t}-J_{b, t}$, in the same way define $\bar{X}_{t}^{\prime b}=X_{t}^{\prime}-J_{b, t}^{\prime}$, and set

$$
Q_{b, n}=\sum\left(\bar{X}_{n k}^{b}\right)^{2}, \quad R_{b, n}=\sum \bar{X}_{n k}^{b} \bar{X}_{n k}^{\prime b}
$$

and $Q_{n}=Q_{\infty, n}$ and $R_{n}=R_{\infty, n}$. For $b>0$ fixed, we will show that $\left\{Q_{b, n}\right\}$ and $\left\{R_{b, n}\right\}$ are reversed martingale sequences. For $\left\{Q_{b, n}\right\}$ this may be established by observing that for some $k, Q_{b, n}-Q_{b, n+1}=2 \bar{X}_{n+1, k}^{b} \bar{X}_{n+1, k+1}^{b}$, and that the $\sigma$-field determined by $Q_{b, n+1}, Q_{b, n+2}, \ldots$ is contained in the $\sigma$-field, $B$, determined by $\bar{X}_{t}^{b}, t \leqq t_{n+1, k}$, and by $\left|\bar{X}_{t}^{b}-\bar{X}_{t^{\prime}}^{b}\right|, t, t^{\prime} \geqq t_{n+1, k}$. Since this $\sigma$-field places a symmetric condition on the symmetrically distributed process on $\left[t_{n+1, k}, t_{n+1, k+1}\right)$, 


$$
E\left(\bar{X}_{n+1, k}^{b} \bar{X}_{n+1, k+1}^{b} \mid \bigotimes\right) \stackrel{\text { a.s. }}{=} \bar{X}_{n+1, k}^{b} E\left(\bar{X}_{n+1, k+1}^{b} \mid \text { \&) } \stackrel{\text { a.s. }}{=} 0\right.
$$

thus

$$
E\left(Q_{b, n}-Q_{b, n+1} \mid Q_{b, n+1,} Q_{b, n+2}, \cdots\right)=0 \quad \text { a.s. }
$$

The assertion about the $R_{b, n}$ sequence follows by a similar but more involved argument. Now

$$
E Q_{b, n}=\sum E\left(\bar{X}_{n k}^{b}\right)^{2}=E\left(\bar{X}_{\left[t_{1}, t_{2}\right)}^{b 2}\right)<\infty
$$

Since the $Q_{b, n}$ are nonnegative, the martingale convergence theorem applies and $Q_{b, n} \rightarrow Q_{b}$ a.s. where $Q_{b}$ is finite. Similarly,

$$
E\left(R_{b, n}\right)^{2}=\sum E^{2}\left(\bar{X}_{n k}^{b}\right)^{2}
$$

and since $\max \left\{E\left(\bar{X}_{n k}^{b}\right)^{2} ; 1 \leqq k \leqq n\right\} \rightarrow 0$ as $n \rightarrow \infty, E\left(R_{b, n}\right)^{2} \rightarrow 0$. It follows by the martingale convergence theorem that $R_{b, n} \rightarrow 0$ a.s. as $n \rightarrow \infty$.

Almost all sample functions are bounded, hence have bounded increments, and if $C_{b}=\left[\left|X_{t}\right| \leqq b / 2\right.$ and $\left.\left|X_{t}^{\prime}\right| \leqq b / 2, t \in T\right]$, then $C_{b} \uparrow C$ as $b \uparrow \infty$ with $P C=1$, and on $C_{b}, R_{b, n}=R_{n}$ and $Q_{b, n}=Q_{n}$ for all $n$. It follows easily that $R_{n} \rightarrow 0$ a.s. and $Q_{n} \rightarrow Q$ finite a.s.

2. If $X_{T}$ is not symmetrically distributed, we then consider the sum $R_{n}=\sum\left(X_{n k}-Y_{n k}\right)\left(X_{n k}^{\prime}-Y_{n k}^{\prime}\right)$, where $X_{T}, Y_{T}, X_{T}^{\prime}$ and $Y_{T}^{\prime}$ are independent and identically distributed. Then by part 1 above, $R_{n} \rightarrow 0$ a.s. and, by Lemma 3, $\sum X_{n k}\left(X_{n k}^{\prime}-Y_{n k}^{\prime}\right) \rightarrow 0$ a.s. and $\sum X_{n k} X_{n k}^{\prime} \rightarrow 0$ a.s. Also, by part 1 above $\sum\left(X_{n k}-X_{n k}^{\prime}\right)^{2}$ converges a.s., and thus we obtain that $\sum X_{n k}^{2}+\sum X_{n k}^{\prime 2}$ converges a.s. By Lemma $4, \sum X_{n k}^{2}$ converges to (some) $S$. It remains to determine the random variable $S$.

3. Let $\pm \epsilon$ be continuity points of $\Psi_{t_{2}}$, set $S_{\epsilon, n}=\sum\left(X_{n k}^{(e)}\right)^{2}, S_{n}=S_{\infty, n}$ and observe that $S_{n}=Q_{n}$. Since almost all sample functions have only a finite number of jumps $\left\{J_{t}\right\}$ in $T$ in absolute magnitude greater than $\epsilon>0$ and none equal to $\pm \epsilon$, and since right and left limits exist, it follows that

$$
S_{n}-S_{\epsilon, n} \stackrel{\text { a.s. }}{\longrightarrow} \sum_{\left|J_{t}\right|>\epsilon} J_{t}^{2}
$$

Thus $S_{\epsilon, n} \rightarrow S_{\epsilon}$ a.s., and as $\epsilon \downarrow 0$, the $S_{\epsilon}$ are nonincreasing. If we set $S_{0}=\lim _{\epsilon \downarrow 0} S_{\epsilon}$, we have $S-S_{0}=\sum J_{t}^{2}$ a.s. It remains to determine $S_{0}$. If the process is symmetrically distributed,

$$
\operatorname{Var}\left(S_{\epsilon, n}\right)=\sum \operatorname{Var}\left(X_{n k}^{(\epsilon)}\right)^{2} \leqq \sum E\left(X_{n k}^{(\epsilon)}\right)^{4} \leqq \epsilon^{2} \sum E\left(X_{n k}^{(\epsilon)}\right)^{2}=\epsilon^{2} E S_{\epsilon, n}
$$

and 


$$
\Psi(\epsilon)-\Psi(-\epsilon) \leftarrow \sum E \frac{\left(X_{n k}^{(\epsilon)}\right)^{2}}{1+\left(X_{n k}^{(\epsilon)}\right)^{2}} \leqq E S_{\epsilon, n} \leqq\left(1+\epsilon^{2}\right) \sum E \frac{\left(X_{n k}^{(\epsilon)}\right)^{2}}{1+\left(X_{n k}^{(\epsilon)}\right)^{2}} .
$$

These inequalities yield uniform integrability of the $S_{\epsilon, n}$, hence $E S_{0}$ $=\lim _{\epsilon \downarrow 0}\{\Psi(\epsilon)-\Psi(-\epsilon)\}=\sigma^{2}$. The inequalities also imply the degeneracy of $S_{0}$ at a constant. If the process is not symmetric, then by part 2 above, $\sum\left(X_{n k}^{(e)}\right)^{2}+\sum\left(X_{n k}^{\prime(e)}\right)^{2} \rightarrow 2 \sigma^{2}$ a.s. as $n \rightarrow \infty$ and then $\epsilon \downarrow 0$. Then Lemma $4 \mathrm{im}-$ plies $\sum\left(X_{n k}^{(e)}\right)^{2} \rightarrow \sigma^{2}$ a.s. in the iterated limit. The theorem is proved.

THEOREM 2. Let $g$ be a continuous function on the real line having a second derivative at 0 and with $g(0)=0$. Then if $X_{T}$ satisfies the conditions of Theorem 1 ,

$$
\int_{T} g\left(d X_{t}\right)=\lim _{n \rightarrow \infty} \sum g\left(X_{n k}\right)=g^{\prime}(0) X_{\left[t_{1}, t_{2}\right)}+\frac{1}{2} g^{\prime \prime}(0) \sigma^{2}+\sum\left\{g\left(J_{t}\right)-g^{\prime}(0) J_{t}\right\}
$$

where, as before, $\sigma^{2}$ is the variance of the normal part of $X_{\left[t_{1}, t_{2}\right)}$ and the sum on the right is over the jumps of $X_{T}$.

Proof. The hypothesis implies that $g$ is of the form $g(x)=a x+b x^{2}+h(x)$, where $h$ is a continuous function and $h(x)=o\left(x^{2}\right)$ at the origin. Because of Theorem 1 we may restrict our attention of the function $h$. We will show that $\sum h\left(X_{n k}\right) \rightarrow \sum h\left(J_{t}\right)$ a.s. In fact,

$$
\left|\sum h\left(X_{n k}^{(e)}\right)\right| \leqq \sup \left\{h(x) / x^{2} ;|x| \leqq \epsilon\right\} \sum\left(X_{n k}^{(\epsilon)}\right)^{2},
$$

and, letting $n \rightarrow \infty$ and then $\epsilon \downarrow 0$, we have $\sum h\left(X_{n \mathbf{k}}^{(e)}\right) \rightarrow 0$ a.s. Then, taking $\pm \epsilon$ to be continuity points of $\Psi_{t_{2}}$,

$$
\sum h\left(X_{n k}\right)-\sum h\left(X_{n k}^{(\epsilon)}\right)=\sum_{\left\{k ;\left|X_{n k}\right|>\epsilon\right\}} h\left(X_{n k}\right)
$$

which converges a.s. to

$$
\sum_{\left|J_{t}\right|>\epsilon} h\left(J_{t}\right)
$$

The proof is completed by letting $\epsilon \downarrow 0$.

\section{REFERENCES}

1. M. Loève, Probability theory, New York, D. Van Nostrand, 1955.

2. - Á l'intérieur du problème central, Publ. Inst. Statist. Univ. Paris (Homage à M. Paul Lévy) vol. 6 (1957) pp. 313-325.

3. Herman Rubin and Howard G. Tucker, Estimating the parameters of a differential process, Ann. Math. Statist. vol. 30 (1959) pp. 641-658.

University of California, Berkeley, California

University of California, Riverside, CAlifornia 